INTERNATIONAL JOURNAL OF SYSTEMATIC BACTERIOLOGY

Vol. 17, No. 3 July 1967

Copyright 1967 by the Iowa State University Press

pp. $231-233$

\title{
THE STATUS OF THE GENUS MICROCOCCUS
}

T. Gibson

Department of General Microbiology

Edinburgh School of Agriculture

Edinburgh, Scotland

SUMMARY. The genus Micrococcus has had an irregular history, and thereis a need for the designation, with international approval, of a neotype strain for the type species, M. luteus. The only strain which has been proposed for designation is ATCC 398. The genus Micrococcus has not yet been clearly distinguisbed from Staphylococcus. The capacity to grow anaerobically at the expense of glucose fermentation shows a gradual transition in a series of strains and lacks precision as a criterion for differentiation between these two genera.

The genus Micrococcus has presented taxonomic problems since it was proposed in 1872 by Cohn for bacteria which are spherical, or nearly so, and nonmotile. In this genus Cohn included species of short rods, such as $\mathrm{M}$. prodigiosus, in which motility was probably overlooked, and also species which may have been true cocci. Subsequently, rod forms were removed, and the genus came into common use as a taxon of coccal bacteria. There has been universal agreement that $M$. luteus is the only species among those proposed by Cohn which could be accepted to be the type species of Micrococcus as this genus is now described. The original characterization of $M$. luteus contains only two definite attributes: (1) the production of a water-insoluble yellow pigment and (2) the formation of a heavy growth in 2 solution containing ammonium tartrate as the sole source of carbon and nitrogen. There appear to have been no later reports of a coccus having these properties except for the statements by Breed and Hucker (1957) concerning strain 398 in the American Type Culture Collection (ATCC). 
Others who have examined this same strain have consistently failed to induce it to grow in Cohn's solution or to utilize tartrate when the solution was supplemented with yeast extract or other addenda. Moreover, in attempts to isolate a coccus capable of developing on ammonium tartrate agar, the only yellow bacterial colonies to appear have been those of rod forms (Gibson 1953).

M. luteus as described by Cohn is clearly a very questionable entity. Several emendations of the original characterization of this species have been put forward, all omitting mention of tartrate utilization, but their relative legitimacy and priority have been disputed. A solution to this dilemma might be the designation, accepted internationally, of a neotype strain of $\underline{M}$. luteus. Thereby, not only this species but also the genus Micrococcus would be established. In view of the irregular history of this case, the designation of the neotype strain would appear to require an Opinion issued by the Judicial Commission. Breed and Hucker (1957) recommended that ATCC 398, which they identified as $\underline{M}$. luteus, be designated as the "type culture" for the genus Micrococcus. This recommendation has received wide publicity, including mention in Bergey's Manual, and no objections have been published. It may be inferred that no other known strain is considered to have a closer resemblance to the original type of $\underline{M}$. luteus. ATCC 398 thus remains the only candidate for designation as the neotype strain for $\underline{M}$. luteus.

The differentiation of Staphylococcus and Micrococcus has been a recurrent problem. While $\underline{\text { S. aureus and the }}$ strictly aerobic micrococci differ widely in their properties, they are connected by a series of intermediate forms which occur, for example, in foods, on skin and in laboratory air. Following the proposal by Evans et al. (1955) to allocate to Staphylococcus the strains that utilize glucose as a source of energy for anaerobic growth, a test for this property has been used for distinguishing the two genera. Yet, with some strains it gives ambiguous or variable results despite precautions to standardize the method. In a recent examination of this test (unpublished) the medium recommended by the Subcommittee on Taxonomy of Staphylococci and Micrococci (1965) proved to be one of the most favourable for the anaerobic growth of the majority of the organisms. But in any medium employed, there were strains which produced so little growth and acid in the absence of oxygen that the results were difficult to judge. A small change in the medium, 
as in its $\mathrm{pH}$ value, could suppress their anaerobic growth completely. That these organisms were indeed capable of producing some macroscopic growth in the absence of oxygen was confirmed by the use of media containing ascorbic acid and reduced resazurin and by incubating cultures in anaerobic jars containing an atmosphere of $\mathrm{H}_{2}+\mathrm{CO}_{2}$. The conclusions reached were (1) that anaerobic growth at the expense of glucose fermentation is a property showing a gradual transition from obvious to absent in a series of strains, and (2) that any arbitrarily chosen standard would be difficult to use because a weak growth might not be invariably reproducible in practice.

For those who take the standpoint that species, and certainly genera, should be distinguishable by several unrelated properties, the genus Micrococcus is poorly defined. The more recent work, such as that on the base composition of deoxyribonucleic acid, has not yet given a guide on the relationships of strains which, on the evidence of other criteria, appear to be intermediate between Micrococcus and Staphylococcus.

\section{REFERENCES}

Breed, R.S. and G.J. Hucker. 1957. The status of the genus Micrococcus Cohn, 1872. Int. Bull. Bact. Nomen. Taxon. 7:113-116.

Evans, J. B., W. L. Bradford, Jr. and C. F. Niven, Jr. 1955. Comments concerning the taxonomy of the genera Micrococcus and Staphylococcus. Int. Bull. Bact. Nomen. Taxon. 5:61-66.

Gibson, T. 1953. The status of the generic names Micrococcus and Staphylococcus and of the species name Staphylococcus aureus. Int. Bull. Bact. Nomen. Taxon. 3:129-133.

Subcommittee on Taxonomy of Staphylococci and Micrococci. 1965. Recommendations. Int. Bull. Bact. Nomen. Taxon. 15:109-110. 